\title{
APE: Average potential evaluation software for the LAB-8 system
}

\author{
DONALD I. TEPAS, GARY KRESS, and ROGER L. KLINGAMAN \\ Saint Louis University, St. Louis, Missouri 63103
}

\begin{abstract}
A paper-tape software system is described which allows the addition and/or subtraction of signal averaging data from the DEC advanced averager program. This pooled averaging data may be plotted, printed, or punched. A DEC LAB-8 system with $4 \mathrm{~K}$ memory and high-speed reader/punch are required. Several examples of the application of the system to human evoked brain response data are presented, and the application of the system to other problems is discussed.
\end{abstract}

Although the signal averaging technique is a popular computer application in contemporary neurophysiological research, many investigators continue to evaluate the results of signal averaging using quite conventional methods which predate the development of modern electronic data processing systems. Thus, many investigators read out the product of their digital signal averaging on an analog $X-Y$ plotter. The resulting analog waveforms are then examined and studied by the investigator in a visual search for deflections which may be related to the variable under study. The cautious investigator, using this traditional approach, then follows this period of examination and study with an effort to measure the amplitude or latency of selected waveform deflections using a ruler or scale of some sort placed on the analog plot. This conventional approach to the product of signal averaging has many advantages. The investigator is able to examine visually the data from many samples at one time. The data are similar in form to that collected and reported in the literature prior to the introduction of the digital computer to the neurophysiological laboratory. The required core memory is minimal, and the peripherals used are relatively cheap and familiar.

On the negative side, however, the manual measurement of analog data plots has a number of clear disadvantages. Obviously, the resolving power of the best ruler does not begin to approach that of decimal values obtained from digital memory storage. Deflections cannot be identified for measurement in analog plots unless the signal-to-noise conditions are such that the deflections can be reliably detected. In most cases, this requires the investigator to average fairly large blocks of data at one time. The time required to gather such blocks may make it difficult for the experimenter to study phenomena having a relatively short time course. Pooling of data across sessions in digital form may require more expensive peripherals. Finally, the comparison of waveforms resulting from different conditions with regard to possible differences in shape becomes a very subjective exercise which frequently promotes disagreement among investigators.

It has been argued that electronic data processing techniques are successfully introduced into the laboratory when they are easy to understand and conventional in output (Tepas, 1974). The software system described utilizes that strategy. APE (average potential evaluation) is a software system that attempts to provide the investigator with the intuitive advantages of conventional analog waveform displays while providing the precision of decimal waveform pooling and measurement with minimal hardware investment. It increases the flexibility and versatility of the signal averaging technique by allowing the investigator to digitally add or subtract waveforms collected at different times. Thus, digitized averages call be pooled to allow detection and reliable measurement of deflections which are difficult or impossible to identify prior to pooling.

\section{HARDWARE REQUIREMENTS}

The computer system required to utilize all of the software reported here is a Digital Equipment Corporation (DEC) LAB-8 contiguration using a PDP-8/I processor with a $4 \mathrm{~K}$ core memory and the AX08 laboratory peripheral. The system includes the DEC high-speed perforated tape read/punch Model PC-8I, an ASR33 Teletype, a Tektronix Type 502A oscilloscope, and a Hewlett-Packard Model 7035B $X-Y$ plotter. No special interfacing is required for this minimal LAB-8 contiguration.

A major section of the software, designated APE II, requires only a PDP-8 computer equipped with standard high-speed read/punch and a Teletype. This section has been run on PDP-8/E, PDP-8/1, and $\mathrm{PDP}-8 / \mathrm{S}$ processors with success. Since the control of timing and the core map of various LAB-8 configurations do not appear to be identical, direct application of the remaining software sections may be limited. 


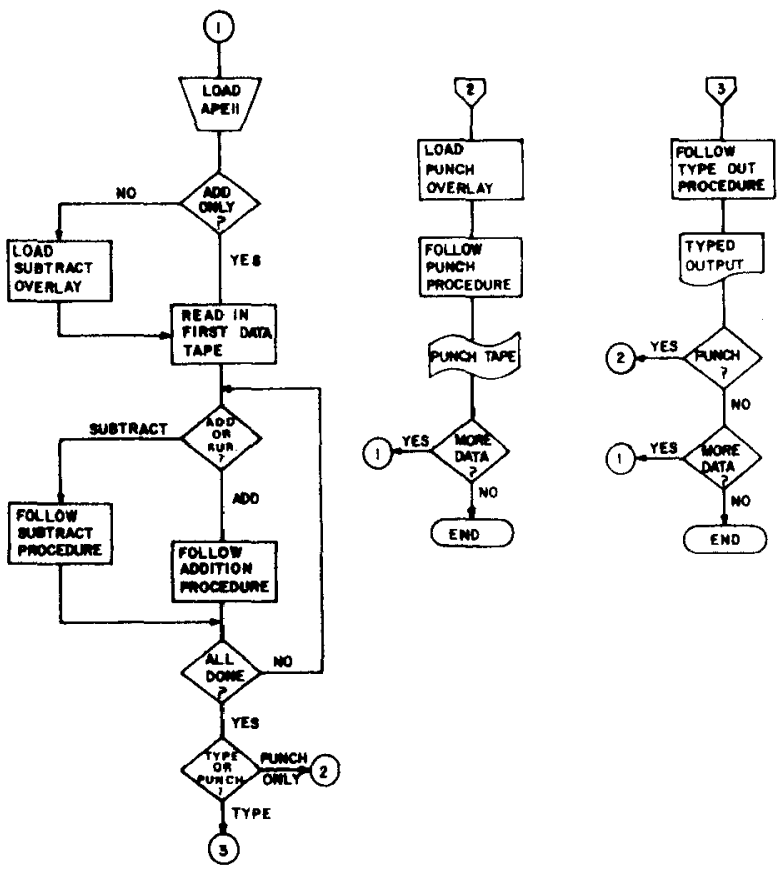

Figure 1. Flow charts showing the use of APE II, the subtract overlay, and the punch overlay.

\section{SOFTWARE}

APE is written in PAL-III assembly language. APE is used in conjunction with the DEC advanced averager program (DEC-LB-U18C-PB) and control tapes prepared by DEC-LB-U17C-PB. A punch paper tape overlay in standard DEC read in mode (RIM) allows the experimenter to dump averaged data using the high-speed paper tape punch once averaging is completed via Section 3 of the advanced averager program. Sections 4 and 5 of the advanced averager are never used. The data tape (DT) produced by this dump is in binary format with two rows of punches representing the binary sum for a particular signal average time point. Thus, the length of this DT is a function of the number of data points (time points) included in the averaging epoch. An experienced operator can produce a DT for most data point epoch sampling rates in a shorter period of time than that required to write out a comparable analog X-Y plot.

Briefly stated, APE provides the investigator with a means of studying DTs computed at different points in experimentation without any loss in precision or the use of additional hardware. These DTs can be added or subtracted from each other. The resulting pooled data can be dumped to produce a new DT, displayed on the scope. X-Y plotted, and/or printed as signed decimal values on the ASR33. These operations are accomplished using the principal program APE II as well as overlays for APE II and the advanced averager program.

Figure 1 is a flow chart for the use of APE II and its two related overlays. APE II is a binary tape loaded into memory by means of the standard DEC binary loader (BIN). Once APE II has been loaded, a DT is placed in the high-speed reader, where it will be read when $\triangle P E ~ I !$ is run. Once the first DT has been read in by APE II, the operator may type out the data or choose to add additional DTs by making appropriate suitch register options. DTs may be subtracted from DT's already added to memory by loading a subtract overlay. Once the desired additions and/or subtractions have been completed, the resulting data can be dumped. producing a DT, by loading the punch overlay. Both of these overlays are binary tapes loaded into memory with BIN. APE II, subtract, and punch are all loaded using the high-speed reader. Paper tapes must be loaded and operations performed in the order indicated in the flow chart.

The advanced averager program software is used to provide the conventional signal averaging data displays for APE punch DTs or DTs dumped lollowing averaging with Section 3 of the advanced averager. Figure 2 is a flow chart for this operation. Using the appropriate control tape, the advanced averager program is read in and a "dummy" average is run to initialize the advanced averager. Following these operations, the APE plot overlay is read in through the high-speed reader using the advanced

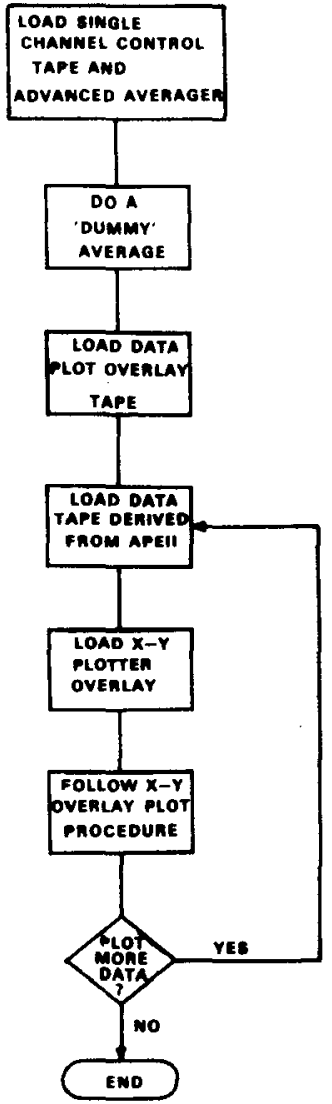

Figure 2. Flow chart showing the use of the plot overlay for reloading data tapes into Section 3 of the advanced averager program allowing scope display and analog X-Y plotting. 


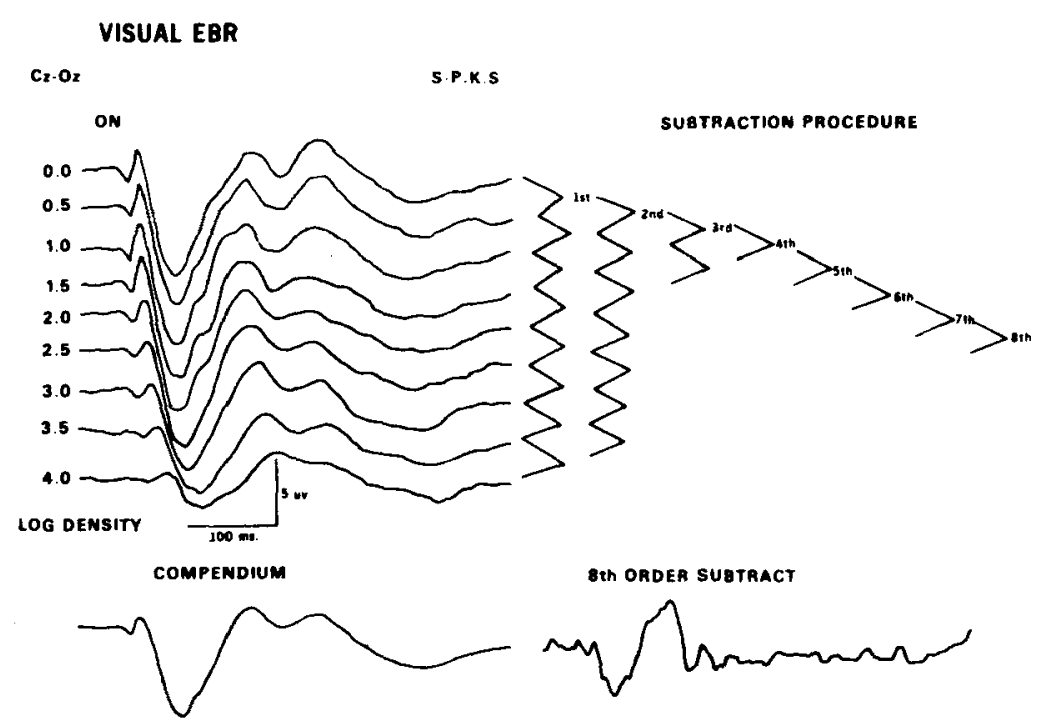

Figure 3. Pooled waveforms produced from the human visual evoked brain response data of Dinges (1973).

averager CONTROL P Teletype operation. A DT is then placed in the high-speed reader, and the program is run using the appropriate starting address. This reads the DT in and allows the display of the pooled waveform in the usual manner. As shown in Figure 2, we obtain our X-Y analog plots using another overlay which allows plotting via Section 3 without use of Sections 4 and 5 of the advanced averager program.

\section{EXAMPLES OF APPLICATIONS}

The APE software system has been used in our laboratory for the evaluation of human and bird evoked brain response (EBR) data obtained by signal averaging of the electroencephalogram (EEG). The complete package has been in use for over 2 years, and the success of this approach is best demonstrated through a number of specific examples. In discussing these applications, the data analysis strategies which have evolved from the use of APE will be highlighted rather than the empirical results, which belong in more appropriate forums.

Previous research from our laboratory has demonstrated that EBR variability need not be correlated with EEG variability (Tepas, Guiteras, \& Klingaman, 1974). Much of the apparent variability in EBR waveforms seems to disappear when sample size is increased by pooling waveforms across sessions. Figure 3 demonstrates this with waveforms produced from the data of Dinges (1973) using APE. In this study, the independent variable was visual stimulus luminance. The EBR waveforms presented in the upper left portion of the figure were obtained by pooling the data from one subject by luminance level across sessions. Each of the nine waveforms is a product of 1,000 tlash onsets. The smooth appearance of the waveforms and their systematic change with luminance is not related to excessive biological amplitier filtering. Waveforms from a single trial within a session are much less regular, and luminance sensitivity is not as evident.

It is important to note that EBR waveform deflection measurements are not made from these analog X-Y plots but rather from the APE Teletype decinal printouts of data points. The $X-Y$ plots are used to assist the investigator in his search for deflection values in the APE printout of pooled data. The identification of reliable deflections can be further enhanced by pooling data independent of stinulus condition. We have termed these waveforms "compendiums." Thus, the compendium waveform shown in the lower left corner of Figure 3 is the sum of the nine waveforms above it and is the product of 9,000 tlash onsets of various luminance levels. The right hall of the figure shows a subtraction procedure applied to the nine waveforms. Such higher order subtraction procedures may reveal which portions of the waveform vary most with changes in the independent variable. Inspection of pooled waveforms such as these, followed by deflection measurement from decimal printout values, dramatically improves deflection identitication and improves the resolution of EBR relationships in many cases.

The appropriate addition and subtraction of waveforms can also be used to provide an initial verification of differences in waveform which are probably related to physiological mechanisms. Figure 4 demonstrates this with waveforms produced from the data of Schweitzer and Tepas (1974) using APE. In this study, the independent variable was auditory stimulus sound pressure level, and differences in ON and OFF EBR waveforms were demonstrated. The waveforms in Figure 4 were obtained by pooling the data from three subjects, each tested in 12 sessions. Each of the ON (top) and the OFF (middle) waveforms is a product of 2,520 auditory presentations or removals. Again, note the 
AUDITORY THREE SS POOLED

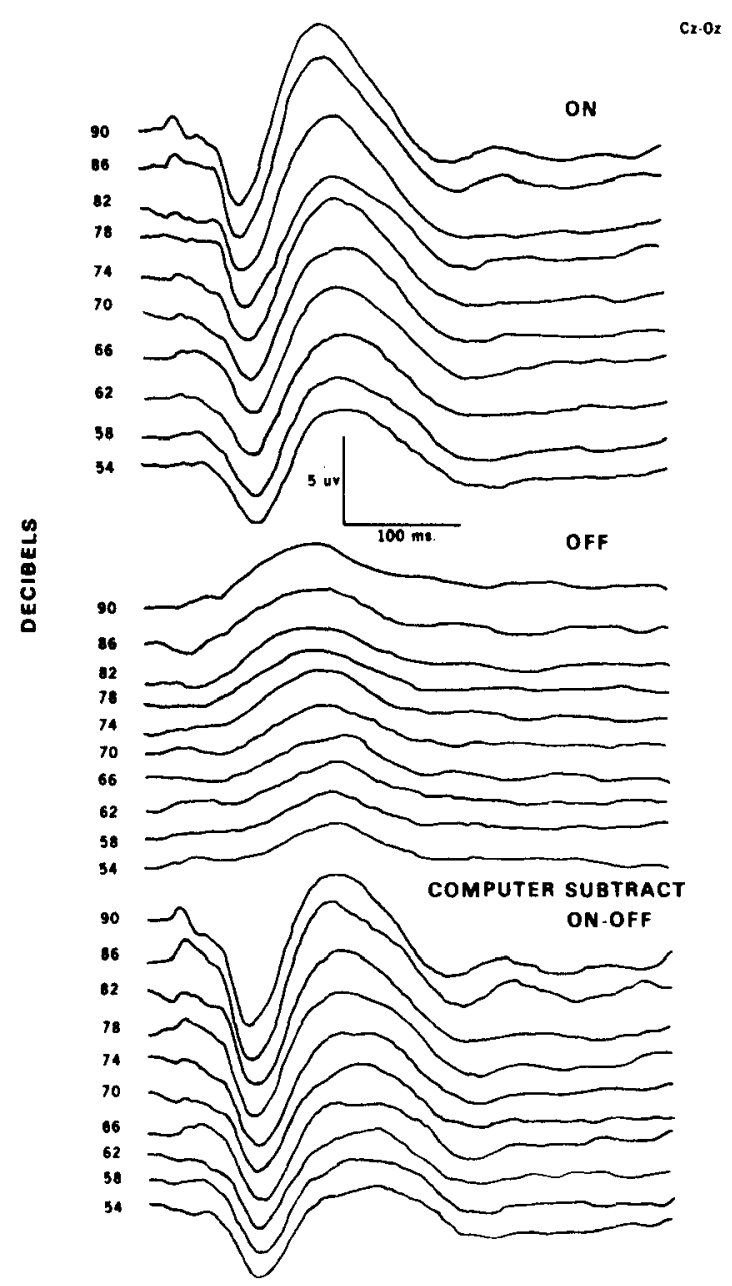

Figure 4. Pooled waveforms produced from the human auditory evoked brain response data of Schweitzer and Tepas (1974).

smooth appearance of the waveforms and their systematic change with sound pressure level. The waveforms at the bottom of the figure were obtained by subtracting the OFF waveforms in the middle of the tigure from their corresponding $\mathrm{ON}$ waveforms. The computer subtract clearly reflects differences in amplitude and latency evident from visual exanination of the $\mathrm{ON}$ and $\mathrm{OFF}$ waveforms. Although the differences are quite obvious in this case. it seems reasonable to suggest that a similar data analysis strategy might be used to initially verify more subtle differences.

It is again important to note that final EBR waveform deflection measurements should be made using the APE Teletype printout as a source for data values. Using this approach, the Schweitzer and Tepas (1974) data reveal a significant difference in EBR intensity function slope as well as the more obvious differences in amplitude and latency. It is important to note that the clear and measurable presence of a deflection in pooled waveforms for all independent variable conditions does not necessarily indicate that the deflection varies as a function of independent variable properties. This is frequently not true. The investigator must not only identify consistently present deflections but also determine which deflections vary systematically with experimental manipulations.

APE soliware can also be used to separate or combine EBR waveforms from different modalities. It appears that. to a considerable degree, evoked potentials summate in a simple algebraic manner in single-channel EEG recordings. This allows the experimenter to perform a sort of computer dissection and or synthesis using APE software. This is demonstrated in Figure 5, which contains waveforms from two separate studies utilizing quite similar data analysis strategies. Both studies involved paired auditory and visual stimulation as well as stimulation in each of the modalities separately. For the pooled waveforms in the left half of Figure 5, the paired stimulation involved simultaneous presentation, while the pooled waveforms in the right half of the figure involved paired stimulation in which the auditory presentation occurred $200 \mathrm{msec}$ following the visual presentation.

For each set of data, the top waveform is that obtained with visual stimulation only (VEBR). The second waveform from the top is that obtained with auditory stimulation only (AEBR). The third waveform is that obtained with paired auditory and visual stimulation (AVEBR). The fourth waveform is a computer synthesis of the third waveform obtained by adding the first two waveforms together. The fifth and sixth waveforms are computer dissections obtained by subtracting the first and second uaveforms. respectively, from the third waveform from the top (AVEBR). This figure provides a representative example of the remarkable degree to which computer synthesis and dissection produces waveforms similar to those produced directly by the appropriate sensory stimulation. The demonstration is particularly impressive for the simultaneous stimulation condition shown in the left half of Figure 5 since the two-modality stimulation waveform (AVEBR) suggests a single response.

\section{DISCUSSION}

Although the examples of application presented have all involved the analysis of human EBR data, the software can obviously be used for the evaluation of any signal averaging data which might be collected using a LAB-8 system. The use of decimal printout data for tinal data analysis is stressed, but it should be noted that the availability of X-Y plots of the data is important in that it makes the experimenter comfortable with what he is doing and at the same time eases the job of searching printout tables for the 
NO DELAY

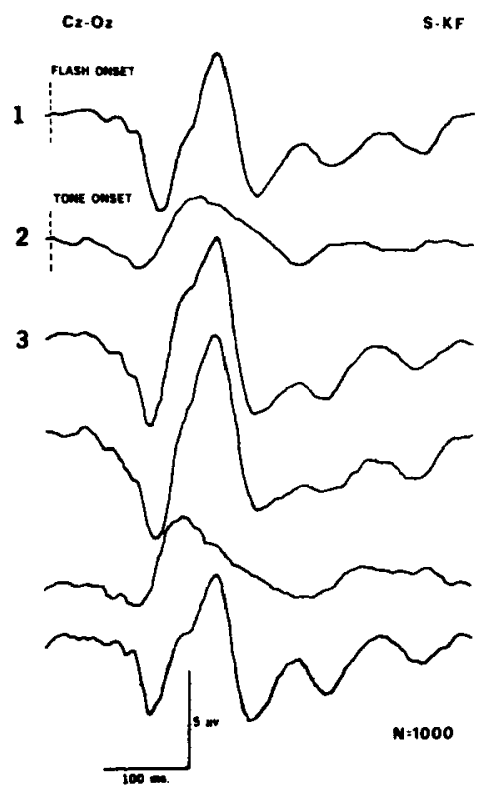

200 Msec. OELAY

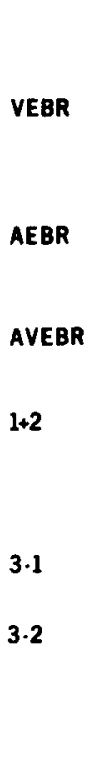

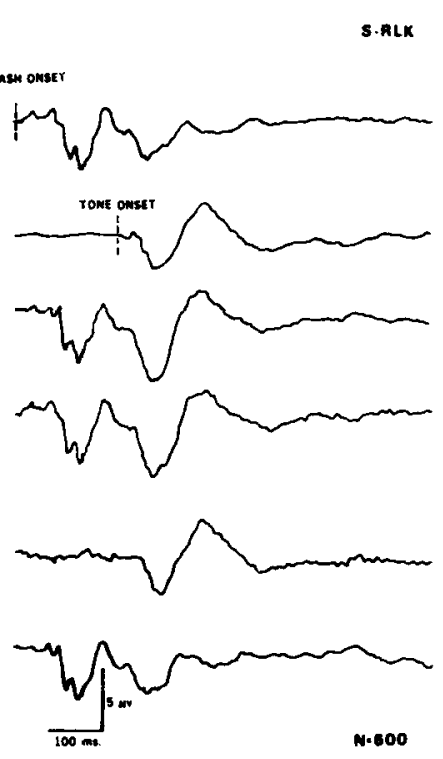

Figure 5. Computer dissection and synthesis of waveforms from the pooled responses to paired auditory and visual stimulation. The data in the left half of the figure are from Tepas, Schweitzer, Walsh, Dinges, and Flanagan (Note 1). The data in the right half are from Klingaman, Dinges, and Kress (Note 2). proper values. It should also be acknowledged that the simple idea of adding and/or subtracting response measurements is not a new one. Most efforts of this sort, however, do not involve the adding and/or subtracting of entire waveforms. This feature is quite desirable in that it improves signal-to-noise conditions as well as promotes a clearer understanding of waveform dynamics.

While other signal averaging systems may have some of the APE evaluation features, a complete evaluation system of this sort for the LAB-8 system using only $4 \mathrm{~K}$ of core memory has not been previously reported in the literature to our knowledge. APE has some obvious disadvantages. The reliance on the advanced averager program is a serious one which will limit APE's direct use. The use of the slow ASR33 for the printout of data significantly slows down data processing. Finally, the general disadvantages of any punch-paper-tape-oriented system must be recognized. Tape defects, high-speed reader error, and the cost of punch paper tape, in the long run, are all problems. In practice, however, the challenges and the simplicity of the APE data analysis strategies developed to date appear to overcome these disadvantages quite well.

\section{REFERENCE NOTES}

1. Tepas, D. I., Schweitzer, P. K., Walsh, J. K., Dinges, D. F., \& Flanagan, K. Human evoked brain responses to simultaneous auditory and visual stimulation. Paper presented at the meeting of the Psychonomic Society, Boston, 1974.

2. Klingaman, R. L., Dinges, D. F., \& Kress, G. Paired auditory and visual responses: Evidence for neural interaction? Paper presented at the meeting of the American Psychological Association. Montreal. 1973.

\section{REFERENCES}

Dinges, D. F. Human visual evoked cortical potentials to stimulus onset and offset. Unpublished masters thesis, Saint Louis University, St. Louis, Missouri, 1973.

Schweitzer, P. K., \& Tepas, D. I. Intensity effects of the auditory evoked brain response to stimulus onset and cessation. Perception \& Psychophysics, 1974, 16, 396-400.

Tepas, D. I. Computer analysis of the electroencephalogram: Evoking, promoting, and provoking. Behavior Research Methods \& Instrumentution, 1974, 6, 95-110.

Tepas, D. I., Guiteras, V. L., \& Klingaman, R. L. Variability of the human average evoked brain response to visual stimulation: A warning. Electroencephalography \& Clinical Neurophysiology. 1974, 36, 533-537. 